\title{
AN IMPROVED PDR INDOOR LOCAION ALGORITHM BASED ON PROBABILISTIC CONSTRAINTS
}

\author{
Yiming You, Ting Zhang, Ye Liu, Yuefeng Lu*, Xiaorong Chu, Chen Feng, Shuo Liu \\ Shandong University of Technology, School of Civil and Architecture Engineering, 255000 Zibo, China- \\ yflu@sdut.edu.cn
}

Commission V, WG V/4

KEY WORDS: Multi-target Encounter, PDR, Indoor Pedestrian Positioning, Probabilistic Constraints, Indoor Network, Key Landmark

\begin{abstract}
:
In this paper, we proposed an indoor pedestrian positioning method which is probabilistic constrained by "multi-target encounter" when the initial position is known. The method is based on the Pedestrian Dead Reckoning (PDR) method. According to the PDR method of positioning error size and indoor road network structure, the buffer distance is determined reasonably and the buffer centering on the PDR location is generated. At the same time, key nodes are selected based on indoor network. In the premise of knowing the distance between multiple key nodes, the forward distance of pedestrians which entered from different nodes can be calculated and then we sum their distances and compared with the known distance between the key nodes, which determines whether pedestrians meet. When pedestrians meet, each two are seen as a cluster. The algorithm determines whether the range of the intersection of the buffer meet the conditions. When the condition is satisfied, the centre of the intersection area is taken as the pedestrian position. At the same time, based on the angle mutation of pedestrian which caused by the special structure of the indoor staircase, the pedestrian's location is matched to the real location of the key landmark (staircase). Then the cumulative error of the PDR method is eliminated. The method can locate more than one person at the same time, as long as you know the true location of a person, you can also know everyone's real location in the same cluster and efficiently achieve indoor pedestrian positioning.
\end{abstract}

\section{INTRODUCTION}

With the rapid development of positioning technology, people have put forward higher and higher requirements of positioning. Seventy percent of the people's daily activities are indoors, so the indoor positioning has been the most pressing demand for modern people. In recent years, there are more and more researches which are closely related with indoor positioning technology, such as the application of ultrasonic positioning technology (Priyantha, 2000), but the ultrasonic attenuation is obvious, and the cost is expensive. Based on $\mathrm{WiFi}$ positioning technology (Kawaguchi, 2009), but because of the complex indoor environment and diverse interference, the accuracy of positioning is not high. It is difficult to be widely used in the daily life. Based on the RSSI range study (Noh, 2013) the signal which is received by the positioning system is also unpredictable due to the uncertainty of the environment. Bluetooth-based positioning technologies (Munoz-Mari, 2007) are only suitable for small-scale positioning and still rely on external signal. Besides, the costs what are spent on the layouts of the signal source infrastructure and maintenance are also higher.

The above positioning methods cannot be used in large-scale due to all kinds of reasons. And single positioning method is difficult to meet people's positioning needs. In this case, some researchers propose a fusion of Bluetooth and map method (Zhao, 2016). Although it improves the accuracy, it still has to rely on external equipment, universality is not strong.
The popularity of smart phones brings people a new way of thinking. Low-cost and fast positioning technology has become the pursuit of many researchers in recent years. How to rely on a mobile phone for high-precision positioning is an urgent problem need to be solved. In the circumstances, Pedestrian dead reckoning (PDR) method (Park, 2016) makes use of only a smart phone, without the need for other ancillary equipment, to carry out indoor pedestrian positioning.

However, single PDR positioning method in short distance, positioning accuracy of which is acceptable. With the increase of distance, the cumulative error increases. Some other problems also exist such as inaccurate positioning, low positioning speed. It is difficult to meet the indoor positioning needs. Therefore, based on the PDR algorithm, this paper presents a "multi-target encounter" probability constraint indoor positioning algorithm. To a certain extent, it can achieve multitarget simultaneous positioning, and effectively reduce the rate of error accumulation. It aims to achieve a high efficiency indoor positioning, and position better.

\section{PEDESTRIAN DEAD RECKONING}

For the main error of PDR positioning method comes from the error of step size and angle, resulting in increased cumulative error (Zhou, 2014). According to the stature and the number of steps, we can determine the step size, the regression equation as the formula (1) shows ( $\mathrm{Li}, 1999)$ :

* Corresponding author 


$$
H=132+0.54 l
$$

Where $H=$ pedestrian height $l=$ pedestrian step length

Because of the limit of the built-in sensor accuracy of the mobile phone, and in order to prevent the accumulation of error of the forward distance obtained by the secondary integration of the acceleration, the pedestrian forward distance is calculated as:

$$
L=n \times l
$$

Where $L=$ the distance forward $n=$ the number of steps

When pedestrian take the $\mathrm{n}$-step is coordinates as:

$$
\begin{aligned}
& X_{\mathrm{n}}=X_{0}+l \sin \theta \\
& Y_{\mathrm{n}}=Y_{0}+l \cos \theta
\end{aligned}
$$

Where $\quad X_{0}, Y_{0}=$ initial coordinates $X_{\mathrm{n}}, Y_{\mathrm{n}}=$ n-step coordinates $\theta=$ azimuth

As shown in Figure 1.

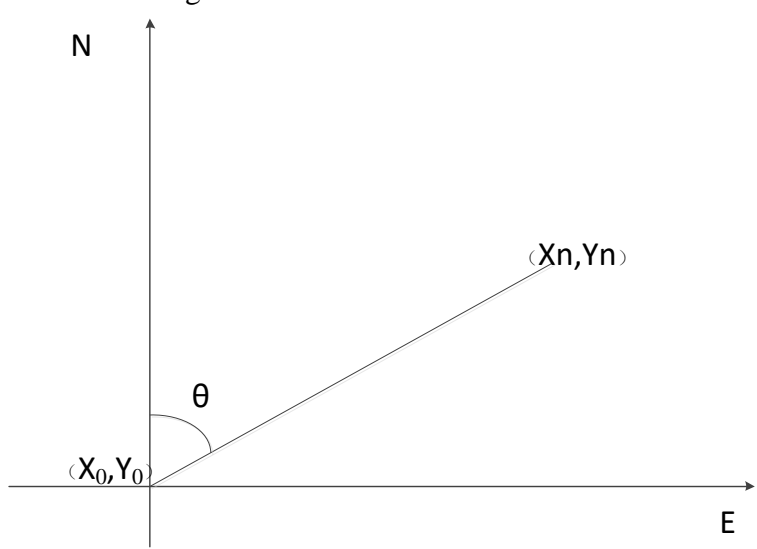

Figure 1. PDR schematic diagram

Since there is a lot of noise in the original acceleration data, in order to correctly detect the number of steps, we use the Chebyshev low-pass filter for denoising. We apply the peak detection algorithm (Mladenov, 2009) to avoid the error of step detection. No. 1 experimenter walked straight forward some 15 steps, as shown in Figure 2.
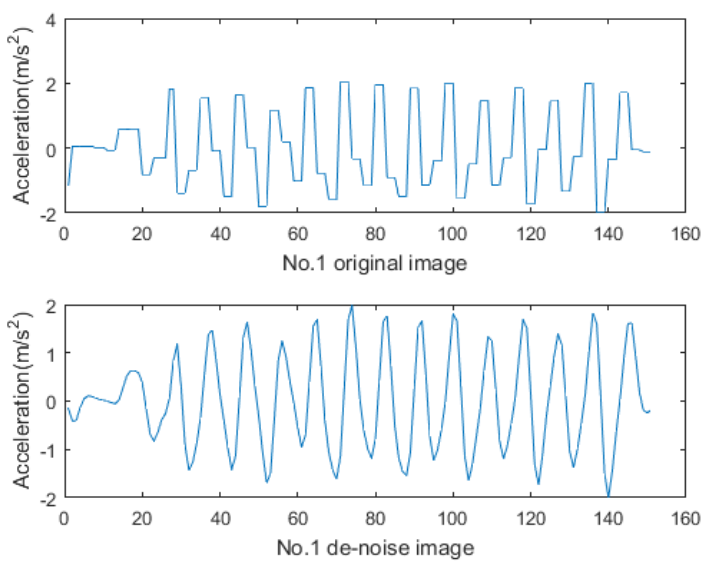

Figure 2. Gait analysis of No. 1 experimenter

No. 1 experimenter's peak detection is 15 steps, which belong to accurate detection.

No. 2 experimenter went straight and then made a turn for 22 steps in total, as shown in Figure 3.
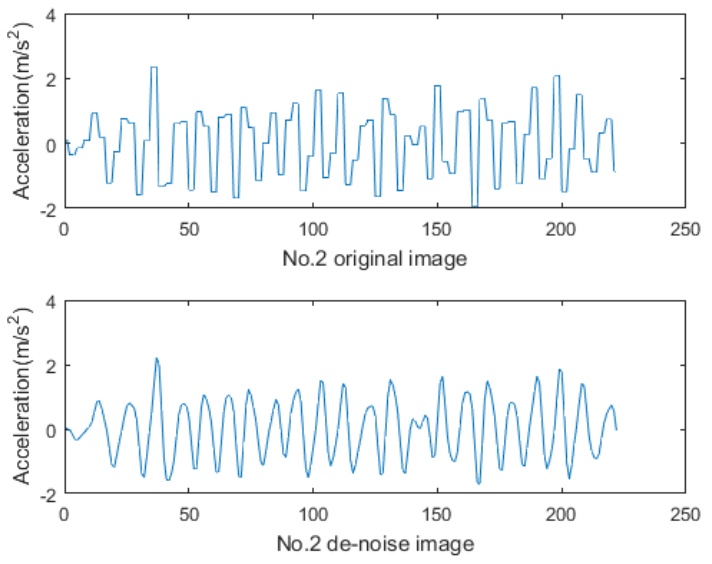

Figure 3. Gait analysis of No. 2 experimenter

For No. 2 experimenter, it detects 23 peaks which means 23 steps. There is one more step than the actual it is. That is because the performer shakes at the corner which causes an added peak.

No. 3 experimenter went straight 14 steps, and the detection can be shown as Figure 4: 

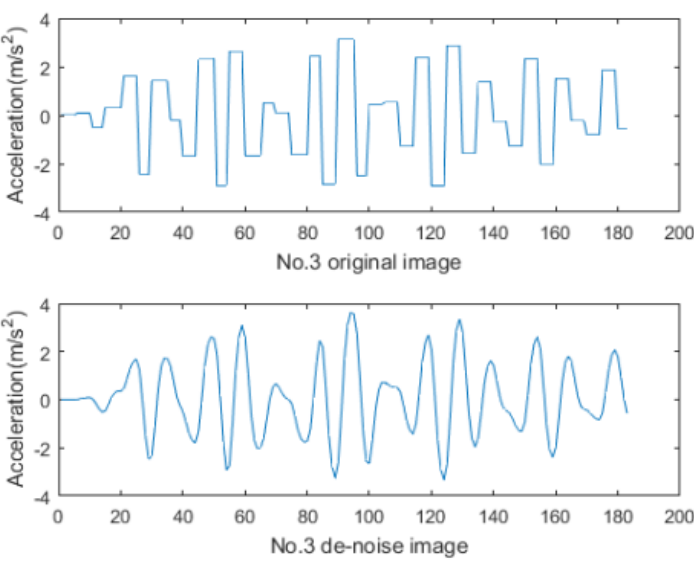

Figure 4. Gait analysis of No. 3 experimenter

For No.3 experimenter. As the Figure 4 shows peak detected 14 steps, its results coincide with the actual situation.

\section{MULTI-TARGET ENCOUNTER}

\subsection{Probability constraint}

The outdoor positioning initial position serves as a known starting position for the positioning target. Indoor positioning can be studied under the condition of knowing initial position and indoor road network.

The experimenter can be tracked according to "multi-target encounter" probability constraint method under the condition that the entrance is one-way street and has known forward direction.

Based on the principle of PDR, forward direction can be detected when the entrance has several roads, as shown in Figure 5.

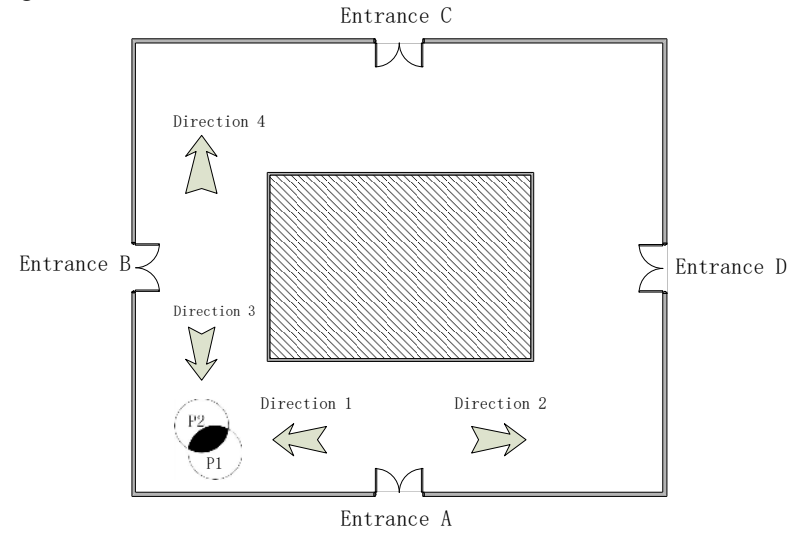

Figure 5. Probabilistic constraints for multi - target positioning

According to the network structure, the entrance and corners are selected as the key nodes, and the distance of each node is known. Take initial position of entrance of the building as the first node, the distance is recorded according to the principle PDR method. The condition is that A, B are two entrances and it has more than one subject. There is no doubt that person $\mathrm{P}_{1}$ heads to the direction 1 and $\mathrm{P}_{2}$ heads to the direction 3 . The forward direction is $1,2,3,4$.
According to the PDR method of positioning error size and indoor road network structure, the buffer distance is determined reasonably and the buffer centring on the PDR location is generated. Then we detect whether there is a buffer intersection between $P_{1}$ and $P_{2}$. When the buffers intersect, we calculate the sum of the forward distances of the two persons and the distance between the entrance $\mathrm{A}$ and the entrance $\mathrm{B}$, and determine whether the distance is equal. The formula is shown as following:

$$
\begin{aligned}
& S_{1}+S_{2}<S_{\mathrm{AB}} \\
& S_{1}+S_{2} \geq S_{\mathrm{AB}}
\end{aligned}
$$

Where $\quad S_{1}=$ the forward distance of $\mathrm{P}_{1}$

$S_{2}=$ the forward distance of $\mathrm{P}_{2}$

$S_{\mathrm{AB}}=$ the accessible distance between the entrance A and the entrance $B$

When the sum of $S_{1}$ and $S_{2}$ is less than $S_{A B}$, the means of PDR positioning is used. When the sum of $S_{1}$ and $S_{2}$ is greater than or equal to $\mathrm{S}_{\mathrm{AB}}$, overlap region can be estimated, according to the formula:

$$
\frac{S_{\mathrm{o}}}{S_{\mathrm{P}}}>\frac{1}{2}
$$

Where

$$
\begin{aligned}
& S_{\mathrm{o}}=\text { overlapping region } \\
& S_{\mathrm{p}}=\text { the generated buffer area }
\end{aligned}
$$

The formula (7) shows that the probability that the pedestrian in the intersection area is greater than the probability in the other location.

As long as overlapping buffer is detected, the sum of distances of the pedestrian forward distance and the available distance can be compared, according to (5), (6). Then the formula (7) is used to judge the probability. If it satisfies the requirement, it can locate the geometric centre of the overlapping area.

The positioning flow chart for this paper is shown in Figure 6 .

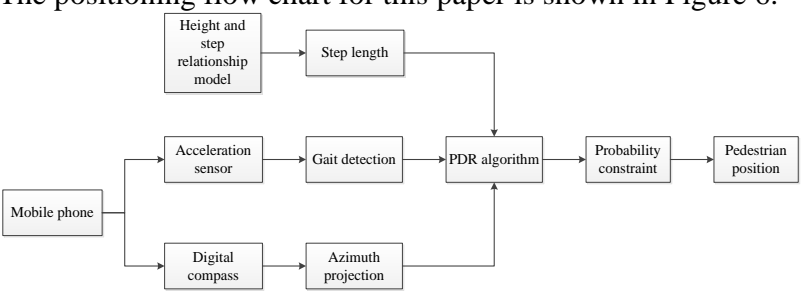

Figure 6. Technical roadmap

\subsection{Indoor map matching constraint}

In order to prevent from positioning exterior space of the building or some non-accessible place, this paper uses the constraints of indoor map, that is, marking the all accessible roads directly. When the PDR positioning drifts to the wall or outside the building, the position can be corrected and be located in the middle of the identified roads again according to the indoor map constraint.

\subsection{Eliminate cumulative errors}

Personal Dead Reckoning (PDR) has little cumulative error in the initial time. With the accumulation of distance, the error increases. In this way, it causes large degree of drift (Jekeli, 
2000) and positioning accuracy is also greatly reduced. The error comes mainly from the azimuth error which caused by the electronic compass susceptibility and the distance error is caused by the acceleration. If you can identify the key indoor landmarks and the location of pedestrians, you can match the location of key landmarks and pedestrians to eliminate the accumulation of errors. In the research of the indoor map, pedestrians seldom have continuous angle reversal behaviour when they go straight in the short distance. When you go up the stairs, it has repeatedly appeared in this behaviour, and the azimuth difference of $180^{\circ}$. As shown in Figure 7. Based on this unconventional change of angle, it is possible to match known points (stairs) based on indoor map constraints, pedestrian's location as well as angle mutations. In this way, the errors that are accumulated before can be eliminated and positioning accuracy can be greatly improved.
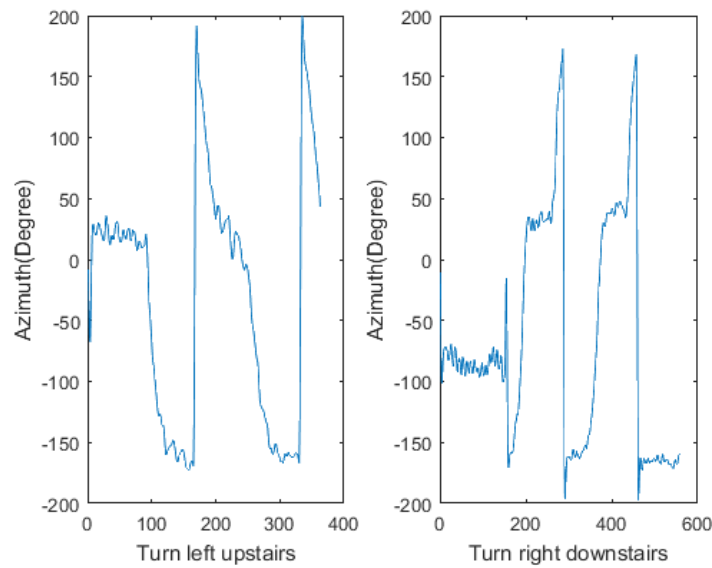

Figure 7. Azimuth changes of up and down

\section{EXPERIMENT AND ANALYSIS}

In order to verify the feasibility of the algorithm, No. 1 experimenter is 170 centimetres $(5 \mathrm{ft} 7 \mathrm{in})$ tall. No. 2 experimenter's height is 167 centimetres $(5 \mathrm{ft} 6 \mathrm{in})$ tall, No. 3 experimenter's height is 153 centimetres ( $4 \mathrm{ft} 7 \mathrm{in}$ ) tall. The experiment is performed by smart phone OnePlus $3 \mathrm{~T}$ with the system of H2OS 2.5 (based on Android 6.0), which is built-in acceleration sensor and electronic compass. Moreover, we select one of the corners of the teaching building as an experimental site.

Experimental behaviour: No. 1 experimenter enters from the entrance $\mathrm{B}$ into teaching building and goes straight. Then No. 1 meets No. 2 and No. 3 experimenters at that time. No. 2 experimenter enters from the entrance $\mathrm{C}$ into and No.3 experimenter from the entrance A into teaching building and walks upstairs. During the experiment, three experimenters lay smart phones flat on their chests when they keep walking. It is shown in the Figure 8.

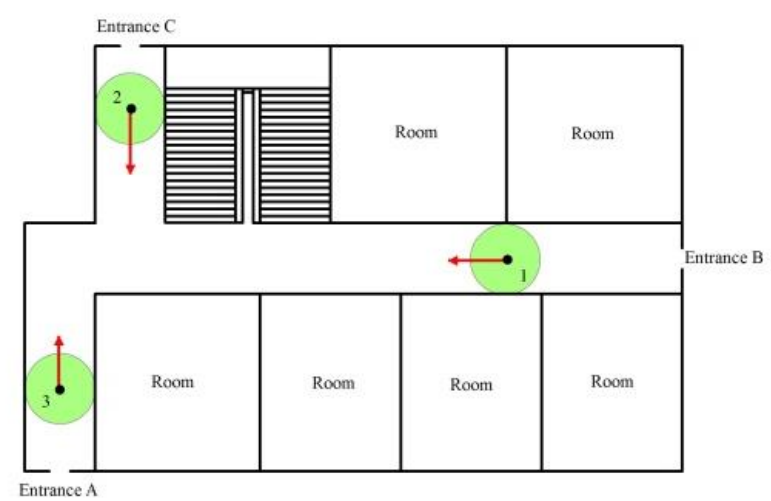

Figure 8. Real map

After a period of time, the No. 1 experimenter and No. 3 experimenters meet each other according to the above algorithm, which is shown in the Figure 9.

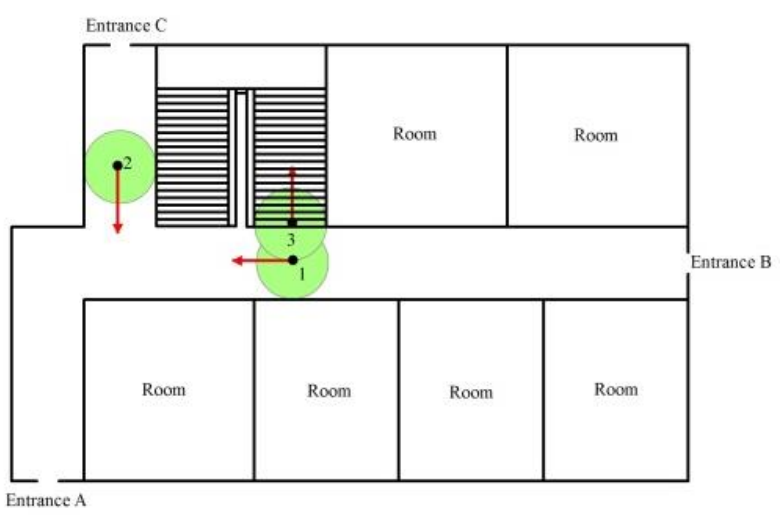

Figure 9. First cluster diagram

At this point, the No. 1 experimenter and the No. 3 experimenter are in the first cluster. We know No.3 experimenter's true position through the angular changes of No. 3 experimenter when he goes upstairs. Then we can get the No. 1 experimenter's real location and eliminate cumulative errors. The situation that No. 2 experimenter enters from the entrance C into teaching building and meets with the No. 1 experimenter, as shown in Figure 10.

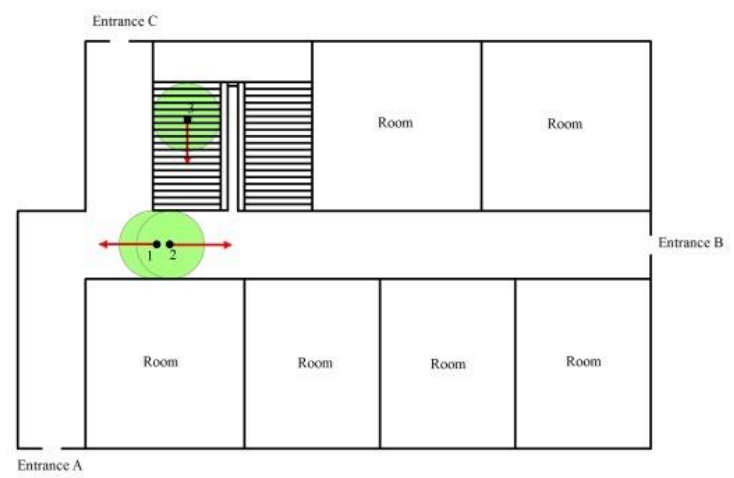

Figure 10. Second cluster diagram

At this point, No. 1 experimenter and No. 2 experimenter are in the second cluster. Because the real position of the No. 1 is 
known, the real position is also the location of the No. 2 experimenter.

\section{CONCLUSIONS}

Through the way of PDR, we can locate the approximate location of indoor pedestrians. The error accumulation problem of PDR method is effectively improved according to the method of probability constraint and the matching of indoor landmarks. Moreover, we can efficiently and accurately locate the true location of other clusters when the true location of one is known. When the crowd is dense, the positioning speed is greatly improved. Experiments have shown that the margin of error is controlled in the range of 2 meter to 3 meters for it has about 1meter reasonable distance among people when they meet. At the same time, there is a time interval detected when pedestrians go upstairs. During this interval, the pedestrian of the first cluster keep on moving for some distance, which results in offset error. But it eliminates the cumulative error of the second cluster. In general, the PDR-based probabilistic constraint method proposed in this paper is fast and stable, and it does not lead to large-scale location errors with the increase of steps.

\section{ACKNOWLEDGEMENTS}

This research has been supported by the Key Laboratory for National Geography State Monitoring (National Administration of Surveying, Mapping and Geoinformation) of China (2016NGCM01), the Shandong Provincial Natural Science Foundation of China (No. ZR2014DL001), the National Natural Science Foundation of China (No. 41501425) and the Key Research and Development Program of Shandong Province (2016GSF122006; 2015GSF122008).

\section{REFERENCES}

Baoding Zhou et al., 2014. User activity awareness assisted indoor pedestrian localization. Geomatics and Information Science of Wuhan University, 39(6), pp.719-723.
Jekeli C., 2000. Inertial navigation systems with geodetic applications. Walter de Gruyte.

Kawaguchi N, Yano M, Ishida S, et al., 2009. Underground positioning: subway information system using WiFi location technology. Tenth International Conference on Mobile Data Management: Systems, Services and MIDDLEWARE. IEEE, pp. 371-372.

Munoz-Mari J, Bruzzone L, Camps-Valls G., 2007. A support vector domain description approach to supervised classification of remote sensing images. IEEE Transactions on Geoscience \& Remote Sensing, 45(8), pp.2683-2692.

Mladenov M, Mock M., 2009. A step counter service for Javaenabled devices using a built-in accelerometer. Cams 09 International Workshop on Context-aware Middleware \& Services: Aff. 1-5.

Noh Y, Yamaguchi H, Iee U, et al., 2013. CLIPS: Infrastructure-free collaborative indoor positioning scheme for time critical team operations. 2013 IEEE International Conference on Pervasive Computing and Communications, pp. 172-178.

Priyantha N B, Chakraborty A, Balakrishnan H.,2000. The cricket location-support system. Proceedings of the 6th Annual Inter-National Conference on Mobile Computing and Networking. New York, NJ, USA: ACM, pp. 32-43.

Park K, Shin H, Cha H., 2013. Smartphone-based pedestrian tracking in indoor corridor environments. Personal and Ubiquitous Computing, 17(2), pp.359-370.

Xiaoping Zhao et al., 2016. An indoor location system based on Bluetooth-corrected and map-matching aided PDR algorithm. Science of Surveying and Mapping, 41(7), pp.53-58.

Yao Li, et al., 1999. Estimation of height based on the regression analysis of step length. Criminal Technology, pp. 171. 